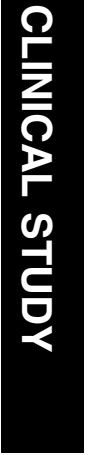

${ }^{1}$ Aravind Eye Care System, Madurai, India

${ }^{2}$ Aravind Eye Care System, Pondicherry, India

${ }^{3}$ F.I. Proctor Foundation, University of California, San Francisco, CA, USA

${ }^{4}$ Department of Microbiology and Immunology, Dartmouth Medical School, Lebanon, $\mathrm{NH}$, USA

${ }^{5}$ Department of Ophthalmology, University of California, San Francisco, CA, USA

${ }^{6}$ Department of Surgery (Ophthalmology), Dartmouth Medical School, Lebanon, NH, USA

${ }^{7}$ Department of Epidemiology \& Biostatistics, University of California, San Francisco, CA, USA

Correspondence: TC Porco, F.I. Proctor Foundation, University of California, Room S351 513 Parnassus Avenue, UCSF, San Francisco,

CA 94143-0412, USA

Tel: + 14154764101 ;

Fax: + 14154760527.

E-mail: travis.porco@ ucsf.edu.

Received: 14 November 2011

Accepted in revised form: 18 April 2012 Published online: 29 June 2012

\section{Predictors of outcome in fungal keratitis}

\begin{abstract}
Purpose To analyse predictors of clinical outcome in fungal keratitis.

Methods Data was collected during a prospective, randomized, controlled, doublemasked clinical trial of treatment for fungal keratitis. Clinical features at presentation and demographics were collected at the enrolment visit for all patients. Pre-specified clinical outcomes included 3-month visual acuity and infiltrate/scar size, time to re-epithelialization, and corneal perforation. A separate multivariable model with each outcome as the dependent variable included all predictor variables.
\end{abstract}

Results Predictors for worse 3-month visual acuity include older age $(P=0.024)$, worse presentation visual acuity $(P<0.001)$, larger infiltrate size at presentation $(P<0.001)$, and pigmented ulcer $(P=0.030)$. Larger infiltrate size at presentation was a significant predictor of worse 3-month infiltrate/scar size $(P<0.001)$. Larger epithelial defect size was a significant predictor of perforation $(P=0.0013)$. Predictors of longer time to re-epithelialization include infiltrate size at presentation $(P<0.001)$ and older age $(P=\mathbf{0 . 0 2 5})$.

Conclusion Ulcer severity at presentation is highly predictive of worse outcomes.

Presentation of clinical characteristics such as baseline acuity and infiltrate scar can provide important information to clinicians about prognosis, and may help guide management and treatment decisions. Prevention of corneal ulcer remains important, as it is difficult to change the course of the ulcer once it has begun.

Eye (2012) 26, 1226-1231; doi:10.1038/eye.2012.99; published online 29 June 2012

Keywords: fungus; keratitis; risk factors

\section{Introduction}

Fungal keratitis is a major cause of visual loss worldwide, especially in tropical climates,
N Venkatesh Prajna', T Krishnan², J Mascarenhas', M Srinivasan ${ }^{1}$, CE Oldenburg ${ }^{3}$, CM Toutain-Kidd ${ }^{4}$, A Sy ${ }^{3}$, SD McLeod ${ }^{3,5}$, ME Zegans S., $^{4}$, NR Acharya ${ }^{3,5}$, TM Lietman ${ }^{3,5,7}$ and TC Porco ${ }^{3,7}$ where as many as half of all corneal ulcers are reported to be fungal in aetiology. ${ }^{1-4}$ Fungal corneal ulcers tend to have worse outcomes than bacterial ulcers; fungal ulcers tend to be more likely to perforate and to require penetrating keratoplasty. 5,6 Fungal infections of the cornea are difficult to treat, and available treatment options are limited. ${ }^{7}$ Commonly recognized risk factors for fungal keratitis include ocular trauma, contact lens use, topical steroid use, ocular surface disease, and history of prior corneal surgery. ${ }^{7,8}$

Better understanding of predictive and prognostic factors in fungal keratitis may improve care. The relationship between potential prognostic factors measurable at the time of diagnosis and important clinical outcomes, such as visual acuity, infiltrate/scar size, time to re-epithelialization of the corneal epithelium, and likelihood of perforation, has not been well characterized. Predictors of clinical outcomes may help guide treatment and management decisions for fungal corneal ulcers. In this report, we analyse several potential predictors of these clinical outcomes for fungal keratitis based on a prospective, randomized, double-masked clinical trial of treatment for fungal corneal ulcers.

\section{Materials and methods}

\section{Clinical trial methods}

The Mycotic Ulcer Treatment Trial Therapeutic Exploratory Study was a randomized, doublemasked clinical trial designed to compare the efficacy of topical natamycin vs topical voriconazole in the treatment of fungal corneal ulcers, either with or without re-scraping of the epithelium. Specific methods for the trial have been described previously. ${ }^{9}$ In brief, 120 patients were randomized to receive either topical natamycin 5\% (Alcon, Fort Worth, TX, USA) or topical voriconazole 1\% (Pfizer, Inc., 
reconstituted by AuroLab, Madurai, India) at the Aravind Eye Care System in Madurai and Pondicherry, India, and independently (in a factorial design) to receive re-scraping of the corneal epithelium at 1 week and 2 weeks after enrolment or not. The primary outcome of the trial was best spectacle-corrected $\log$ MAR visual acuity (BSCVA) at 3 months after enrolment. ${ }^{9}$ Institutional Review Board approval was obtained at the University of California, San Francisco, Dartmouth Medical School, and Aravind Eye Hospital.

Complete demographic and ocular medical history was obtained for all patients enrolled in the trial at the enrolment visit. In addition to the enrolment visit, regular study visits occurred every 3 days until re-epithelialization, at 3 weeks, and at 3 months after enrolment. Infiltrate/scar size, epithelial defect size, hypopyon, and depth measurements, as well as assessment of adverse events, including corneal perforation, occurred at each study visit. BSCVA was measured according to the study protocol at enrolment, 3 weeks, and 3 months. Ulcer location at baseline was determined by photography and was divided into four categories: entirely in the periphery; overlapping the central 4-mm circle and periphery without filling the centre; entirely in the central 4-mm circle; and completely filling the 4-mm circle and extending to the periphery. BSCVA is reported in logMAR units, with approximate Snellen equivalents given (rounded to two significant figures in the denominator).

Three-month BSCVA was compared between natamycin and voriconazole and between the presence and absence of corneal scraping for the primary outcome of the trial, reported elsewhere. ${ }^{9}$ Here, we report the results of a secondary analysis of the following variables (obtained at presentation) for their effects on the ultimate clinical outcome: age (years), gender, visual acuity (logMAR), estimated depth of the infiltrate, infiltrate/ scar size, epithelial defect size, feathery edges, endothelial plaque, hypopyon, location (central/ peripheral), multiple lesions, pigmented, epithelial defect size, history of recent ocular trauma, object of corneal injury (vegetative/non-vegetative), occupation (agriculture/non-agriculture), adnexal infection, corneal or lid abnormality, on topical antifungal at presentation, on topical antibiotic at presentation, on steroids at presentation, and duration of symptoms. Both infiltrate/ scar size and epithelial defect size were taken as the geometric mean of the longest diameter and the maximum distance perpendicular to this diameter. Pre-specified clinical outcomes analysed include BSCVA at 3 weeks and 3 months, infiltrate/scar size at 3 weeks and 3 months, time to re-epithelialization, and corneal perforations.

\section{Statistical methods}

We considered the following four primary outcome variables: BSCVA at 3 months, scar size at 3 months, the time to re-epithelialization, and the presence of corneal perforation or transplant.

Univariate analyses for continuous outcome variables BSCVA and scar size were based on the Wilcoxon rank-sum test when comparing two groups, and monotone regression ${ }^{10}$ when comparing between ordered categories. We assessed the relationship between each baseline continuous variable and each of BSCVA and scar size using linear regression; significance was assessed using a permutation test because of failure of normality.

Univariate analyses for perforation were based on Fisher's exact test (for dichotomous baseline variables), the Cochrane-Armitage test (for ordered categorical variables), and logistic regression (for continuous baseline variables, Wald test). All analyses of reepithelialization times were conducted using Cox proportional hazards regression. We adjusted for multiple comparisons using the conservative Bonferroni-Holm method.

Multivariable analysis was conducted using logistic regression for perforation, Cox proportional hazards regression for re-epithelialization time, and linear regression for 3-month scar size. We modelled the relationship between baseline visual acuity and 3-month visual acuity using GAM (generalized additive models). ${ }^{11}$ We compared a linear relationship to a non-linear smoothing spline using the F-test. ${ }^{12}$ The adequacy of linear regression was assessed using plots of residuals $v$ fitted values. Normality and homoskedasticity were assessed using plots of the residuals against the fitted values (linear regression). We conducted Hosmer-Lemeshow goodness of fit tests for logistic regression, and we tested the adequacy of the proportional hazards assumption using the method of Grambsch and Therneau. ${ }^{13}$ Patients for whom 3-month visual acuity was unavailable through their original cornea because of corneal transplant were assigned a $\log$ MAR score of 1.7 (poorer than 20/800) or their 3-week acuity, whichever was worse. Patients lost to follow-up before 3 weeks were omitted from the analysis.

\section{Results}

One hundred and twenty patients were enrolled in the trial. Median (mean) baseline visual acuity among these patients was logMAR 0.71 (0.93), or approximately 20/100 (20/170), with an interquartile range of $1.28 \log$ MAR. The median (mean) infiltrate or scar size at baseline was $3.57 \mathrm{~mm}(3.93 \mathrm{~mm})$, with an interquartile range of $2.49 \mathrm{~mm}$. 
The median (mean) visual acuity at 3 months was logMAR 0.21 (0.53), or approximately 20/30 (20/70), with an interquartile range of $0.84 \log$ MAR. The median (mean) scar size at 3 months was $3.49 \mathrm{~mm}(4.03 \mathrm{~mm})$, with an interquartile range of $2.67 \mathrm{~mm}$. Nineteen perforations occurred during the course of the trial (15.8\% of enrolled patients) and 15 therapeutic penetrating keratoplasties $(12.5 \%)$ were performed. Finally, the median time to re-epithelialization was 18.5 days, with the minimum at 1 day (95\% confidence interval 14.0 to $>21.0$ days).

A total of 14 patients (11.6\%) had missing acuity measurements at 3 months; no differences between these patients and the overall population with respect to age, gender, or baseline acuity were observed (Wilcoxon ranksum tests or Fisher tests), or for scar size at 3 months. Two patients $(1.7 \%)$ were omitted owing to loss to followup by 3 weeks.

Table 1 shows the univariate analyses of each predictor for each of the four primary outcomes. Important predictors by univariate analysis for all of the outcomes include presentation visual acuity, infiltrate depth, infiltrate/scar size, and epithelial defect size, presence of an endothelial plaque, presence of hypopyon, and central location of the ulcer $(P<0.001)$. Virtually identical results were obtained when a different value (1.9) was assigned as the visual acuity following perforation requiring corneal transplant (showing that the findings are not greatly affected by this choice). Univariate $P$-values that remain statistically significant following BonferroniHolm adjustment are shown in bold in Table 1.

The relationship between visual acuity at presentation and the visual acuity at 3 months is shown in Figure 1. The curvilinear relationship provides a substantially better description of the data than a linear model $(P=0.008, F$-test). Figure 1 also illustrates that most lesions in which baseline and final visual acuity were good (logMAR below 0.4) were small lesions with feathery borders and no hypopyon. Large lesions that fill the central $4 \mathrm{~mm}$ pupil as defined by photography and extend into the periphery frequently correspond to poor initial and final acuity (upper right corner).

Table 2 shows the multivariable models for all predictors for each of the primary outcomes. Significant predictors of worse 3-month visual acuity include older age $(P=0.024)$, worse presentation visual acuity $(P<0.001)$, larger infiltrate size at presentation $(P<0.001)$, and pigmented ulcer $(P=0.037)$. Virtually identical results were obtained when 1.9 was assigned for the visual acuity following corneal transplant.

For multivariable modelling of 3-month infiltrate/scar size, we found that the only significant predictor for worse 3-month infiltrate/scar size was worse infiltrate size at presentation $(P<0.001)$. Larger epithelial defect size was a significant predictor for perforation $(P=0.0013)$. Infiltrate size at presentation $(P<0.001)$ and older age $(P=0.025)$ were significant predictors of longer time to re-epithelialization.

\section{Discussion}

The relationship between baseline clinical and demographic predictors and clinical outcome has not been well characterized for fungal keratitis. Better understanding of these risk factors may help guide clinicians with more accurate prognoses and appropriate treatment. In this study, we have characterized the association between multiple clinical and demographic predictors with four pre-specified clinical outcomes: BSCVA 3 months from enrolment, infiltrate/scar size 3 months from enrolment, perforation, and time to re-epithelialization of the epithelial defect.

Univariate analyses revealed multiple predictors that may be associated with a 3-month visual acuity. When the predictors were analysed in a multivariable model with BSCVA at 3 months as the outcome, presentation visual acuity, presentation infiltrate size, male gender, older age, and pigmented lesion were found to be significant predictors. It is reasonable that a worse presentation visual acuity would be highly predictive of a worse resultant visual acuity. Likewise, a larger presentation infiltrate size would also be expected to predict larger outcome scar size and therefore worse outcome visual acuity.

The demographic factors of older age and male gender were also significant predictors of worse visual outcomes. In addition, older age was a significant predictor of increased time to re-epithelialization (though this would not remain significant following correction for multiple tests). Prior reports on microbial keratitis (bacterial, fungal, and viral) have demonstrated that older patients may have worse prognoses and outcomes compared with younger patients. ${ }^{14-16}$ This association may be the result of reduced immunity in the elderly or different risk factors affecting the population. ${ }^{14}$ Two separate studies in South India have found the microbial keratitis to affect men more than women. ${ }^{3,17}$ In this study, $66 \%$ of the participants were men. Male patients may be more likely to be agricultural workers or involved in outdoor activities, placing them at higher risk of trauma or injury to the cornea. There may be differences in aetiologic organism with different inoculums, placing males at risk for different organisms. If there are differences in outcome between organisms, this could place men at higher risk for poor outcome.

Pigmentation of the ulcer at presentation offers interesting insights into predicting visual outcomes. Macroscopic pigmentation is typically characteristic 


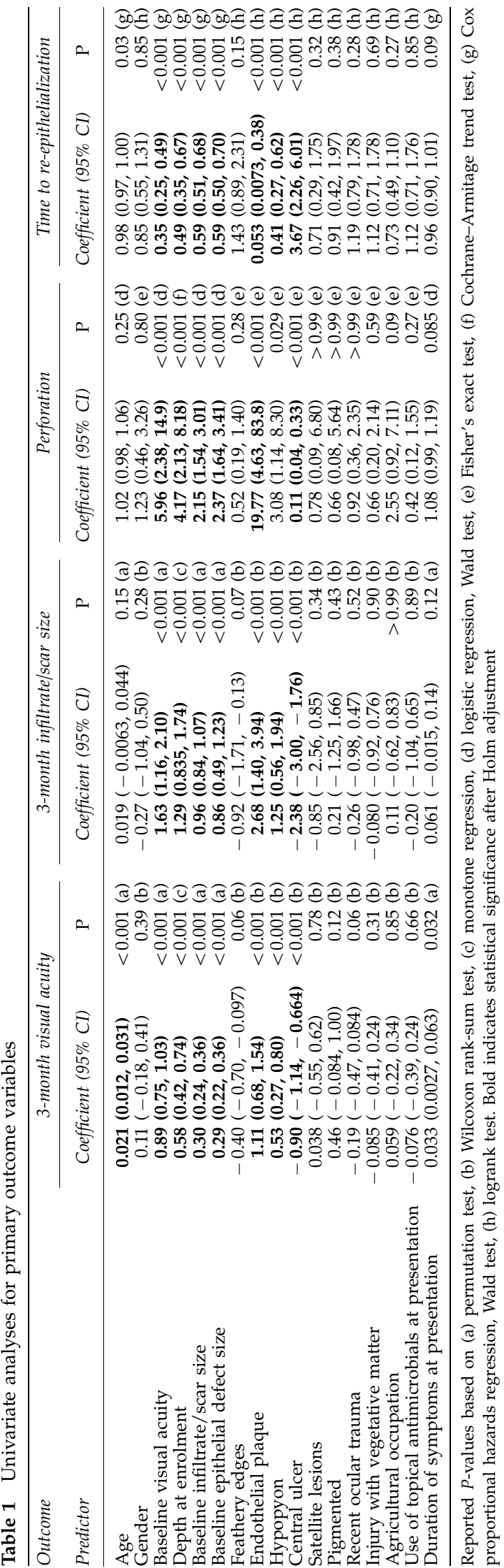

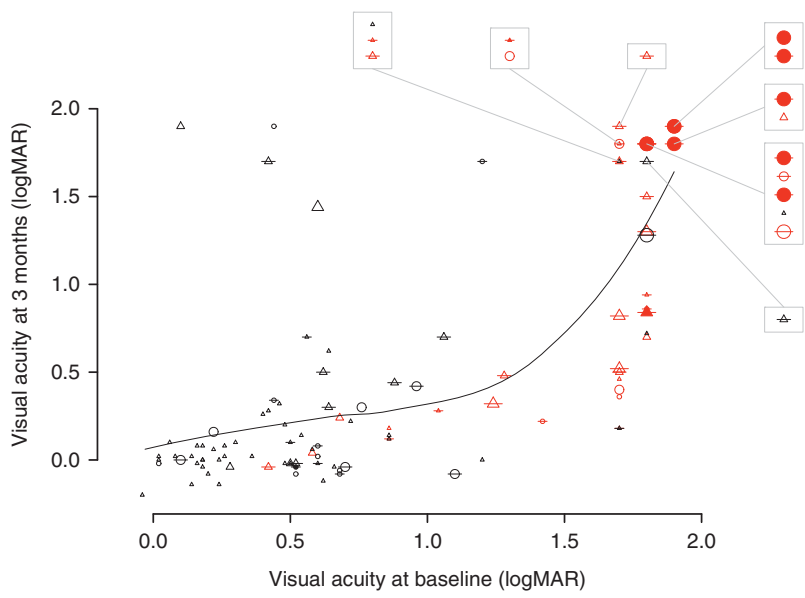

Figure 1 Three-month best spectacle-corrected visual acuity (BSCVA) plotted against the baseline BSCVA. Visual acuity is expressed as logMAR ('count fingers' was expressed as $1.7 \log$ MAR, 'hand motion' as 1.8, and 'light perception' as 1.9). The depth of the ulcer is expressed as the size of the plotting symbol. A triangle indicates a lesion with a feathery border; a circle no feathery border. The presence of endothelial plaque is indicated by a filled symbol. A horizontal bar indicates hypopyon. When the lesion fills the central $4 \mathrm{~mm}$ and extends into the periphery, the plot colour is red. The curve shows a smoothing spline (non-parametric regression) fit. Note that in the upper right of the figure, plot points are superimposed at several locations; the separate symbols for each person at each location are shown in separate breakout boxes to the top and right of the figure, with a line connecting the breakout box to the graphical location where the superimposed symbols broken out within that box appear on the plot.

of dematiaceous fungi, such as Curvularia and Bipolaris spp. Several studies have suggested the use of macroscopic pigmentation as a diagnostic tool for fungal keratitis. ${ }^{18-20}$ These studies have also hypothesized that pigmentation indicates lower virulence and less severe inflammation, and presence of fungal filaments superficially on the anterior stroma. $18,19,21$ Our findings suggest that, in addition to being a diagnostic indicator of fungal keratitis, pigmentation of the corneal ulcer can also be a prognostic indicator for worse visual outcomes, contrary to prior hypotheses. By the time pigmented hyphae are visible on the cornea, the infection may have been present for a longer period of time and therefore has developed into a more severe ulcer. In this study, ulcers that had visible pigmented hyphae in the cornea had significantly longer duration of symptoms before presentation, indicating that these were indeed further progressed ulcers. Visible pigmentation in the cornea may therefore be an indication that the ulcer is further advanced and more likely to have a poor outcome.

In a multivariable analysis with infiltrate/scar size at 3 months as the outcome, presentation infiltrate/scar size 
Table 2 Multivariable analyses for four outcomes: the 3-month visual acuity (generalized additive model), the 3-month scar size (linear regression), the presence of perforation (logistic regression), and the re-epithelialization time (Cox proportional hazards regression)

\begin{tabular}{|c|c|c|c|c|c|c|c|c|}
\hline & \multicolumn{8}{|c|}{ Outcome } \\
\hline & \multicolumn{2}{|c|}{ 3-month visual acuity } & \multicolumn{2}{|c|}{ 3-month scar size } & \multicolumn{2}{|c|}{ Perforation } & \multicolumn{2}{|c|}{ Time to re-epithelialization } \\
\hline & $\begin{array}{c}\text { Coefficient, } \\
95 \% \text { CI }\end{array}$ & $P$ & $\begin{array}{c}\text { Coefficient, } \\
95 \% \text { CI }\end{array}$ & $P$ & $\begin{array}{l}\text { Odds Ratio, } \\
\text { 95\% CI }\end{array}$ & $P$ & $\begin{array}{l}\text { Hazard Ratio, } \\
95 \% \text { CI }\end{array}$ & $P$ \\
\hline \multicolumn{9}{|l|}{ Predictor } \\
\hline Age & $\begin{array}{c}0.00719 \\
(0.00105,0.0133)\end{array}$ & 0.024 & & & & & $\begin{array}{c}0.985 \\
(0.971,0.998)\end{array}$ & 0.025 \\
\hline Gender & $\begin{array}{c}0.170 \\
(0.00362,0.335)\end{array}$ & 0.048 & & & & & $\begin{array}{c}0.687 \\
(0.442,1.07)\end{array}$ & 0.096 \\
\hline Baseline visual acuity & Nonparametric & $<0.001$ & & & & & & \\
\hline Depth at enrollment & $\begin{array}{c}0.121 \\
(-0.0172,0.260)\end{array}$ & 0.089 & & & $\begin{array}{c}2.04 \\
(0.881,4.74)\end{array}$ & 0.10 & & \\
\hline Infiltrate size at baseline & $\begin{array}{c}0.146 \\
(0.0735,0.218)\end{array}$ & $<0.001$ & $\begin{array}{c}0.756 \\
(0.526,0.986)\end{array}$ & $<0.001$ & & & $\begin{array}{c}0.536 \\
(0.444 \quad 0.647)\end{array}$ & $<0.001$ \\
\hline Epithelial defect size at enrollment & & & $\begin{array}{c}0.243 \\
(-0.0119,0.498)\end{array}$ & 0.065 & $\begin{array}{c}2.01 \\
(1.31,3.06)\end{array}$ & 0.0013 & & \\
\hline Feathery edges & & & $\begin{array}{c}-0.306 \\
(-0.728,0.117)\end{array}$ & 0.16 & & & & \\
\hline Location & $\begin{array}{c}0.186 \\
(-0.0789,0.451)\end{array}$ & 0.17 & & & & & & \\
\hline Endothelial plaque & & & & & $\begin{array}{c}7.45 \\
(0.957,57.9)\end{array}$ & 0.055 & $\begin{array}{c}0.173 \\
(0.231,1.30)\end{array}$ & 0.088 \\
\hline Satellite lesions & & & & & $\begin{array}{c}0.0453 \\
(0.00129,1.59)\end{array}$ & 0.088 & & \\
\hline Pigmented & $\begin{array}{c}0.331 \\
(0.0240,0.638)\end{array}$ & 0.036 & & & & & & \\
\hline Injury with vegetative matter & & & $\begin{array}{c}-0.327 \\
(-0.759,0.105)\end{array}$ & 0.14 & & & & \\
\hline
\end{tabular}

Backwards stepwise regression was used in each case, beginning with the following predictors: age, sex, visual acuity at baseline, enrollment depth, baseline infiltrate/scar size, epithelial defect size at baseline, the presence of feathery borders, the presence of endothelial plaque, hypopyon, whether or not the lesion completely fills the central $4 \mathrm{~mm}$ and extends into the periphery or not, recent ocular trauma, injury with vegetable matter, agricultural occupation, use of topical antimicrobial medication at diagnosis, duration of symptoms prior to diagnosis, drug assignment (voriconazole or natamycin), and scraping assignment. A generalized additive model (with a nonparametric smoothing term for baseline visual acuity) was used for the 3-month visual acuity outcome, because univariate regression of 3-month acuity $v$ s baseline acuity showed evidence of a nonlinear relationship.

was found to be a significant predictor. As with 3-month visual acuity, a larger baseline infiltrate/scar size results in a larger 3-month infiltrate/scar size. Additionally, infiltrate size at presentation was a significant predictor of time to re-epithelialization and perforation. Therefore, infiltrate/scar size on presentation was predictive of all our three main clinical outcomes and our adverse outcome of perforation. Size of the ulcer has been shown to be an important predictor of primary treatment failure for fungal keratitis in previous studies. ${ }^{5,22}$ Our analyses show that the size of the ulcer also predicts treatment course, including adverse effects such as perforation, and visual outcomes.

Data for this study were collected as part of a randomized controlled trial, comparing outcomes in patients randomized to receive either topical voriconazole or topical natamycin, and to receive either corneal re-scraping or no re-scraping (in a two by two factorial design). The trial found no significant difference in outcomes between patients randomized to receive voriconazole or natamycin, and a non-significant trend towards worse visual acuity in patients randomized to receive re-scraping. ${ }^{9}$ This analysis was a secondary analysis of the trial, and therefore the study was not specifically designed for these analyses. Exclusion or inclusion criteria may have biased these results.

For example, patients with impending perforation were excluded from the trial, and therefore we did not enrol the most severe cases.

Our finding of presentation visual acuity being predictive of the 3-month visual acuity outcome offers an important application to clinical practice. In clinical settings outside the context of an investigative study, visual acuity is not always measured accurately when patients present with corneal ulcers. If poor baseline vision after careful measurement is identified, physicians caring for such patients may want to use this as an indication to initiate more aggressive treatment and to follow these patients more closely because of their increased risk of complications. More importantly, the prognostic information can be of great value to the patient. Likewise, our finding that macroscopic pigmentation is predictive of worse outcome visual acuity should also allow physicians to offer prompt antifungal treatment and prognostic information.

In the context of our currently limited treatment regimens, our study suggests that it is difficult to change the course of an ulcer; even given proper treatment, an 
ulcer that is severe at presentation is likely to have poor outcomes. Therefore, improved prevention of corneal ulcers is of great importance. However, we expect our model of predictive factors will aid in the development of future treatment algorithms as new treatment options develop, particularly those that allow for more aggressive treatment of fungal keratitis. Having a framework in which physicians are able to use baseline characteristics to predict ulcer severity and outcome will help physicians to continue adapting and improving treatment practices in addition to providing important prognostic information in the presence of appropriate therapy, with the ultimate goal of improving visual outcomes and patient quality of life.

\section{Summary}

\section{What was known before}

- Fungal keratitis is a major cause of vision loss worldwide, and fungal infections of the cornea are difficult to treat.

- Common risk factors include ocular trauma, contact lens use, steroid use, and ocular surface disease.

What this study adds

- Ulcer severity at presentation is highly predictive of worse clinical outcomes.

- Presentation indicators of severity, such as visual acuity or infiltrate size, can provide clinicians with important information about prognosis.

- Once an ulcer presents, it is difficult to substantially change its course, and prevention of corneal ulceration remains important.

\section{Conflict of interest}

The authors declare no conflict of interest.

\section{References}

1 Chowdhary A, Singh K. Spectrum of fungal keratitis in north India. Cornea 2005; 24: 8-15.

2 Leck A, Thomas P, Hagan M, Kaliamurthy J, Ackuaku E, John $\mathrm{M}$ et al. Aetiology of suppurative corneal ulcers in Ghana and south India, and epidemiology of fungal keratitis. Br J Ophthalmol 2002; 86: 1211-1215.

3 Srinivasan M, Gonzales C, George C, Cevallos V, Mascarenhas J, Asokan B et al. Epidemiology and aetiological diagnosis of corneal ulceration in Madurai, south India. Br J Ophthalmol 1997; 81: 965-971.
4 Whitcher J, Srinivasan M, Upadhyay M. Corneal blindness: a global perspective. Bull World Health Organ 2001; 79: 214-221.

5 Miedziak A, Miller M, Rapuano C, Laibson P, Cohen E. Risk factors in microbial keratitis leading to penetrating keratoplasty. Ophthalmology 1999; 106: 1166-1171.

6 Wong T, Ng T, Fong K, Tan D. Risk factors and clinical outcomes between fungal and bacterial keratitis: a comparative study. CLAO J 1997; 23: 275-281.

7 Srinivasan M. Fungal keratitis. Curr Opin Ophthalmol. 2004; 15(4): 321-327.

8 Jurkunas U, Behlau I, Colby K. Fungal keratitis: changing pathogens and risk factors. Cornea 2009; 28(6): 638-643.

9 Prajna N, Mascarenhas J, Krishnan T, Reddy P, Prajna L, Srinivasan $\mathrm{M}$ et al. Comparison of natamycin and voriconazole for the treatment of fungal keratitis. Arch Ophthalmol 2010; 128(6): 672-678.

10 Barlow R, Bartholomew D, Bremner J, Brunk H. Statistical Inference Under Order Restrictions: The Theory and Application of Isotonic Regression. Wiley: New York, 1972.

11 Hastie T, Tibshirani R. Generalized Additive Models. Chapman \& Hall/CRC: New York, 1990.

12 Wood S. Generalized Additive Models: An Introduction Using R. Chapman and Hall: Boca Raton, FL, 2006.

13 Grambsch P, Therneau T. Proportional hazards tests and diagnostics based on weighted residuals. Biometrika 1994; 81(3): 515-526.

14 van der Meulen I, van Rooij J, CP N, Van Cleijnenbreugel H, Geerards A, Remeijer L. Age-related risk factors, culture outcomes, and prognosis in patients admitted with infectious keratitis at two Dutch tertiary referral centers. Cornea 2008; 27(5): 539-544.

15 Morlet N, Minassian D, Butcher J, Group OS. Risk factors for treatment outcome of suspected microbial keratitis. $\mathrm{Br} \mathrm{J}$ Ophthalmol 1999; 83: 1027-1031.

16 Green M, Apel A, Naduvilath T, Stapleton F. Clinical outcomes of keratitis. Clin Exp Ophthalmol 2007; 35 421-426.

17 Gopinathan U, Garg P, Fernandes M, Sharma S, Athmanathan S, Rao G. The epidemiological features and laboratory results of fungal keratitis: a 10-year review at a referral eye care center. Cornea 2002; 21(6): 555-559.

18 Garg P, Gopinathan U, Choudhary K, Rao G. Keratomycosis: Clinical and microbiologic experience with dematiaceous fungi. Ophthalmology 2000; 107: 574-580.

19 Berger S, Katsev D, Mondino B, Pettit T. Macroscopic pigmentation in a dematiaceous fungal keratitis. Cornea 1991; 10: 272-276.

20 Oldenburg C, Prajna N, Lalitha P, Krishnan T, Mascarenhas J, Vaitilingam $\mathrm{C}$ et al. Clinical signs in dematiaceous and hyaline fungal keratitis. Br J Ophthalmol 2011; 95(5): 750-751.

21 Garg P, Vemuganti G, Chatarjee S, Gopinathan U, Rao G. Pigmented plaque presentation of dematiaceous fungal keratitis: a clinicopathologic correlation. Cornea 2004; 23: 571-576.

22 Lalitha P, Prajna N, Kabra A, Mahadevan K, Srinivasan M. Risk factors for treatment outcome in fungal keratitis. Ophthalmology 2006; 113: 526-530. 